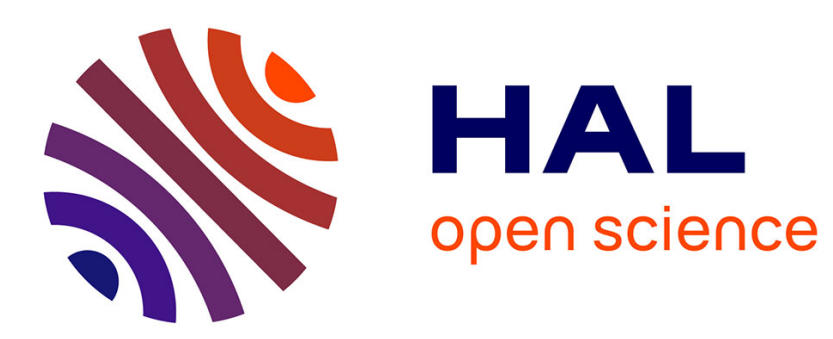

\title{
Dual-reference digital holographic interferometry for analyzing high-density gradients in fluid mechanics
} Jean-Michel Desse, François Olchewsky

\section{To cite this version:}

Jean-Michel Desse, François Olchewsky. Dual-reference digital holographic interferometry for analyzing high-density gradients in fluid mechanics. Optics Letters, 2018, 43 (8), pp.1635-1638. 10.1364/OL.43.001635 . hal-01829355

\section{HAL Id: hal-01829355 \\ https://hal.science/hal-01829355}

Submitted on 4 Jul 2018

HAL is a multi-disciplinary open access archive for the deposit and dissemination of scientific research documents, whether they are published or not. The documents may come from teaching and research institutions in France or abroad, or from public or private research centers.
L'archive ouverte pluridisciplinaire HAL, est destinée au dépôt et à la diffusion de documents scientifiques de niveau recherche, publiés ou non, émanant des établissements d'enseignement et de recherche français ou étrangers, des laboratoires publics ou privés. 


\title{
Dual-reference digital holographic interferometry for analyzing high density gradients in fluid mechanics
}

\author{
JeAn-Michel Desse ${ }^{*}$, François OlCheWsky \\ ONERA, The French Aerospace Lab, and LMFL, Laboratoire de Mécanique des Fluides de Lille - Kampé de Fériet, CNRS FRE 2017, 5 Boulevard \\ Paul Painlevé, BP 21261, 59014 Lille Cedex, France \\ *Corresponding author: Jean-Michel.Desse@onera.fr
}

\begin{abstract}
This paper proposes a dual-reference digital holographic interferometer for analyzing high refractive index encountered in transonic and supersonic flows. For that, a Wollaston prism is inserted in the reference arm in order to simultaneously generate two orthogonally polarized reference waves. As consequence, recorded interferograms contain two crossed and perpendicular interference patterns which give two orders fully separated in the Fourier spectrum. It is then possible to analyze a transparent object regardless the orientation of the refractive index gradient using the two phase maps reconstructed with each of the two first interference orders. Fusion of the phase maps yield a single phase map in which the phase singularities are removed. Experimental results demonstrate the suitability of the proposed approach for analyzing shock waves in the unsteady wake flow around a circular cylinder at Mach 0.75. (C) 2018 Optical Society of America
\end{abstract}

OCIS codes: (090.1995) Digital holography; (090.2880) Holographic interferometry; (110.2650) Fringe analysis; (110.5086) Phase unwrapping.

In the domain of fluid mechanics, the flows consecutive to detachments, marginal vortices, jets and vortex bursting need to be characterized by fine and adapted optical methods. This requirement aims at deepening the phenomenology of these complex flows and to build databases from the gas density field for the qualification of the computational fluid dynamics codes. The two main alternative techniques currently used are velocity measurements that allow either local measurement at very high frame rates (velocity vector measurement by Laser Doppler Anemometry) [1], or full-field measurement with two or three components (Particle Image Velocimetry) [2]. It is therefore crucial to define tools for measuring smaller and smaller data in space and time. The digital holographic interferometer developed and tested at ONERA is a non-intrusive metrological tool, with high spatial and temporal resolution. The setup provides absolute and instantaneous measurements of the full field of gas density and the evolution of the field in time, unlike the differential methods which only give access to the local derivative of the measured quantity (differential interferometry, schlieren techniques, background oriented schlieren). The optical method has been successfully tested for the analysis of subsonic unsteady flows [3,4], but as the Mach number increases and becomes close to the transonic regime, shock waves appear in the flow and cause phase singularities in phase unwrapping which depend on the fringe orientation. In order to solve this problem, we propose a dual reference digital holographic interferometer in which the sensitivity to phase singularities is reduced, and both reference waves interfere with the object wave. The two generated interference patterns have crossed orientations and they are recorded on the same sensor. For the interferogram analysis, 2D Fourier spectrum exhibits two fully separated diffraction orders that provide the reconstruction of two phase maps, one for each interference pattern. As high density gradients are analyzed (shock waves, for instance), phase singularities may appear in the first interference pattern because the fringes are parallel to the shock wave. In this case, the second interference pattern which has the fringes perpendicular to the shock wave is used and the refractive index shift can be determined through the shock wave. Then, fusion of the two phase maps yields a single phase map in which the phase singularities are removed.

In literature, off-axis holographic interferometers generating several references either with several wavelengths or a single wavelength generating a double reference were published. As examples, for the measurement of displacements of scattering object, several authors use different color laser sources to measure the different components of the deformation [5,6], in digital holographic microscopy, to reconstruct the synthetic wavelength phase distribution of a reflective objet [7], or to quantify the threedimensional structure of cells [8]. Others researchers propose to measure the state of polarization of a magnetic sample by using two orthogonally polarized reference beams [9]. Barada et al. [10] and Ochiai et al. [11] describe a single reference wave and two object waves having perpendicular linear polarizations and driven by spatial light modulators. In this case, two cameras are used to record an interference pattern formed between one of the two object waves with the reference wave. Lee et al. propose a dualchannel Mach-Zehnder interferometer which provides the reconstruction of the field object from only two step measurements [12]. More precisely, the nearest works of those described in this letter are those of Abdelsalam et al. who 
developed a dual-wavelength digital holographic set-up based on the polarization separation for imaging polarimetry [13] and specially, the work of Beghuin [14] and Colomb [15] who used two reference beams presenting orthogonal polarizations and different propagation directions to measure spatial variations of the polarization of an object wave. In their experimental setup, they have to use several beam splitters, $\lambda / 4$ and $\lambda / 2$ wave plates and a variable retarder. As comparison with our setup, we have just to introduce a Wollaston prism in the reference beam to generate two reference beams presenting orthogonal polarizations. Finally, we can also mention the work of Tahara [16] who imaged the distribution of the polarization of three-dimensional objects by using the space-division multiplexing of holograms. Unfortunately, the micro-polarizer array device used in this technique limits the resolution by a factor of 2 .

In this letter, we present a dual-reference Mach-Zehnder digital holographic interferometer with phase map fusion for analyzing high refractive index in transonic and supersonic flows. In this type of flow, the difficulty lies in the measurement of phase step consecutive to the high density gradients where, for instance, it is not possible to get continuous interference fringes at the crossing of the shock waves. Thus we propose to use a Wollaston prism in order to simultaneously generate two reference waves with orthogonal polarization. As consequence, the interferogram recorded by the sensor contains two interference patterns with crossed directions which provide two orders fully separated in the Fourier spectrum. It is then possible to analyze a transparent object regardless the orientation of the refractive index gradients using the two phase maps reconstructed with each of the two interference orders. The next paragraph discusses an example of analyzing shock waves in the unsteady wake flow around a circular cylinder at Mach 0.75 .

The interferometer is presented in Fig. 1 and it uses a pulsed laser vertically polarized with an exposure time of 5 nanoseconds at a pulse rate of $10 \mathrm{~Hz}$. The coherence length of the laser is 3 meters.

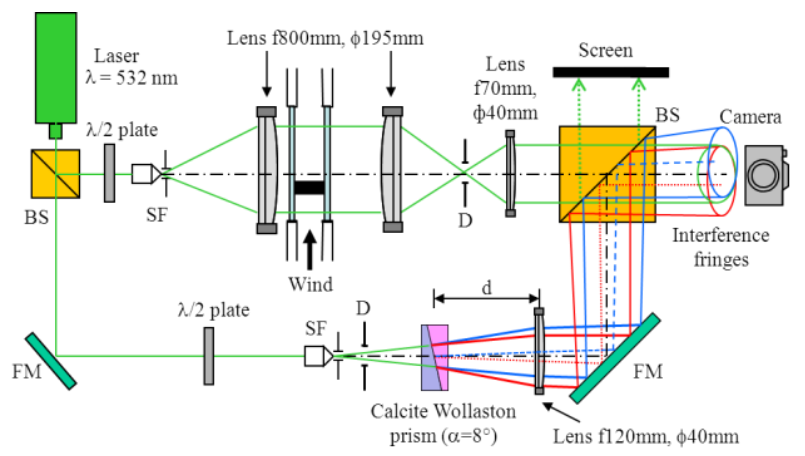

Fig. 1. Dual-reference digital holographic interferometer using Wollaston prism. BS, SF, FM and D are beam splitter cube, spatial filter, flat mirror and diaphragm respectively.

The first beam splitter cube forms the object beam which passes through the test section (object beam) and the reference beam which passes below the test section. In the object beam, a spatial filter associated with the $8800 \mathrm{~mm}$ lens allows illuminating the test section with parallel beam. A second f800 $\mathrm{mm}$ lens associated with the $f 70 \mathrm{~mm}$ lens provides the magnification of the test section at the sensor plane. In the reference beam, a second spatial filter and the $120 \mathrm{~mm}$ lens allow obtaining a parallel beam. A second beam splitter cube recombines the reference and object beams on the sensor. In the reference arm, a Wollaston prism is inserted between the spatial filter and the lens $f=120 \mathrm{~mm}$ at a distance $d$ of the lens. The prism separates the reference wave into two orthogonally polarized waves $\mathrm{S}$ and $\mathrm{P}$ (red and blue in Fig. 1). These two waves are separated by a small birefringence angle $\varepsilon=$ 2. $\left(n_{e}-n_{o}\right) \cdot \tan (\alpha)$ that depends on the pasting angle $\alpha$ and on the material (quartz or calcite) of the prisms. The refractive index variation $n_{e}-n_{o}$ represents the extraordinary and ordinary indices of the material. As the Wollaston prism is located inside the focal length of the f120 mm lens, the magnification $G$ can be obtained according to Eq. (1):

$$
G=\frac{f}{f-d}=\frac{\varepsilon}{\epsilon^{\prime}}
$$

where $\varepsilon^{\prime}$ is the birefringence angle measured behind the $120 \mathrm{~mm}$ lens. It can be calculated as a function of the parameters of the optical setup and of the prism parameters:

$$
\varepsilon^{\prime}=2\left(1-\frac{d}{f}\right) \cdot\left(n_{e}-n_{0}\right) \cdot \tan (\alpha)
$$

The Wollaston prism inside the reference arm generates two reference waves, namely S (red) and P (blue), which can interfere with the $\mathrm{S}$ and $\mathrm{P}$ components of the object wave. Two configurations are possible. If one needs to generate orthogonal interference patterns inclined at $-45^{\circ}$ and $+45^{\circ}$, the polarization direction of the reference and object waves has to be turned by $45^{\circ}$ using a half wave plate oriented at $22.5^{\circ}$ as shown in Fig. 1. Fig. 2 (a) shows the polarization $\mathrm{S}$ and $\mathrm{P}$ of the two reference waves (red and blue, respectively) and the object wave (green). Fig. 2(b) shows the reference waves shifted by $d x=\varepsilon^{\prime} f$ and the location of the object wave with respect to the two reference waves to obtain the inclined fringes patterns. Fig. 2(c) shows the two interferences produced by the $\mathrm{S}$ and $\mathrm{P}$ components of the reference and object waves. Fig. 2(d) shows the result of the superposition of the two patterns recorded by the sensor which gives a checkerboard of interference fringes inclined at $45^{\circ}$.

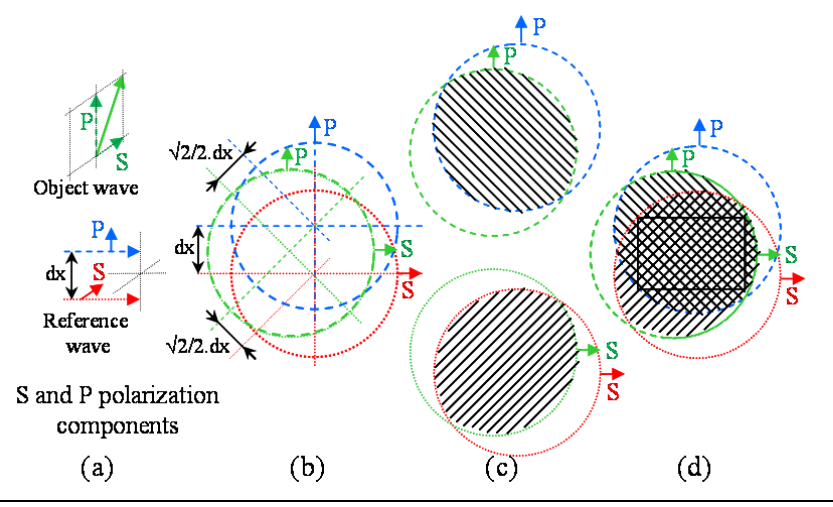

Fig. 2. Scheme of interferences generated at $+45^{\circ}$ and $-45^{\circ}$.

The second configuration consists in obtaining horizontal and vertical fringes. For that, the two half wave plates are removed of the set-up and the Wollaston prism is rotated by $45^{\circ}$ around its axis so that the $\mathrm{S}$ and $\mathrm{P}$ components are inclined at $-45^{\circ}$ and $+45^{\circ}$ of the vertical axis. In the object beam, the polarization direction becomes vertical and the $\mathrm{S}$ and $\mathrm{P}$ components of the object wave are inclined at $-45^{\circ}$ and $+45^{\circ}$ of the vertical axis as shown in Fig. 3(a). Fig. 3(b) and Fig. 3(c) show the location of the object wave 
with respect to the two reference waves to obtain the vertical and horizontal checkerboard of interference fringes. The result of the superposition of the two patterns recorded by the camera (horizontal and vertical checkerboard) is given in Fig. 3(d).

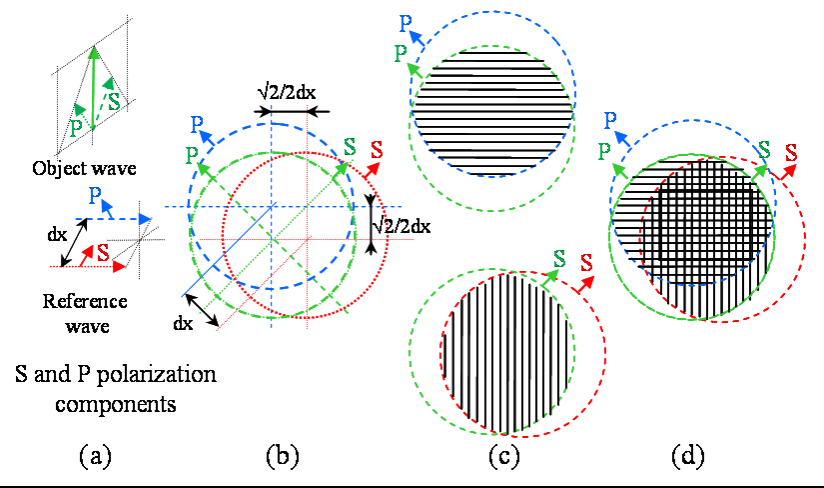

Fig. 3. Scheme of interferences generated at $0^{\circ}$ and $90^{\circ}$.

Then, a very good spatial resolution has to be reached to correctly define the fringe period $i$ ( 5 to 10 pixels, for instance). With the used camera (Hamamatsu Orca flash 2.8, pixel size of $3.65 \mu \mathrm{m}$ ), the angle $\varepsilon^{\prime}$ can be calculated by:

$$
\varepsilon^{\prime}=2 \cdot \arcsin \left(\frac{\lambda}{2 i}\right)
$$

and, if the Wollaston prism is set at a distance $d$ equal to $f / 2$, the angle $\varepsilon$ can be found according to Eq. (1). The numerical application gives $\varepsilon=4.85^{\circ}$ and $9.62^{\circ}$ for $i=10$ pixels and 5 pixels respectively. As the birefringence angle has to be large, this requires the use of a calcite Wollaston $\left(n_{e}-n_{0}=0.172\right)$ and a pasting angle $\alpha$ of $8^{\circ}$ was chosen. In these conditions, when the Wollaston is placed at $d$ varying from $30 \mathrm{~mm}$ to $90 \mathrm{~mm}$, the pixels number of the fringe period varies from 4 to 12 pixels. The dual-reference digital holographic interferometer using Wollaston prism has been tested for analyzing the unsteady wake flow downstream a circular cylinder, $20 \mathrm{~mm}$ in diameter, at transonic Mach number (0.75). Fig. 4 shows the reference interferogram where the enlarged parts of the cylinder exhibits the checkerboard formed by the two inclined pattern of interference fringes arbitrarily set at $+45^{\circ}$ and $-45^{\circ}$ and the measurement interferogram shows the checkerboard disturbed by the separation layers emitted from the upper and lower surfaces.
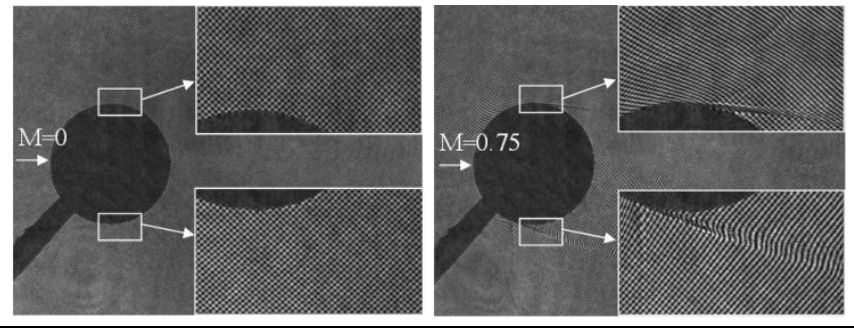

Fig. 4. Interferograms recorded without and with flow.

On each interferogram, we can see the shadow of the tips of pitot pressure and the unsteady pressure sensor which are implemented at the skin of the cylinder. Both sensors pass inside the model and outside the test section. With this equipment, the interferograms which are taken at different instants of the vortex street can be correlated with the unsteady pressure signals.
Then, the 2D Fast Fourier Transform is computed for each interferogram and the results are presented in Fig. 5. The spectra show the zero order at center, the +1 and -1 orders corresponding to the interferences between the polarized reference and object waves S and P (respectively red and blue). The useful signal for reconstructing the phase maps is contained inside the circles. The spatial frequencies of the fringes can be estimated from the spectrum. Thus, the +1 order of the $S$ polarization is located at the coordinates $\left(\mathrm{u}_{\mathrm{s}}=-21.69\right.$ lines $/ \mathrm{mm}, \mathrm{v}_{\mathrm{s}}=19.79$ lines $\left./ \mathrm{mm}\right)$ and the +1 order of the $\mathrm{P}$ polarization at the coordinates $\left(\mathrm{u}_{\mathrm{p}}=18.55\right.$ lines $/ \mathrm{mm}$, $v_{p}=21.12$ lines $/ \mathrm{mm}$ ). From that, the fringe period of the two $S$ and $\mathrm{P}$ patterns along $+45^{\circ}$ and $-45^{\circ}$ axis, respectively, are equal to $34.06 \mu \mathrm{m}$ and $35.57 \mu \mathrm{m}$. The pixel number in each fringe period is 9.33 pixels for $\mathrm{S}$ and 9.75 for $\mathrm{P}$. This was expected by positioning the Wollaston prism at $d=80 \mathrm{~mm}$.
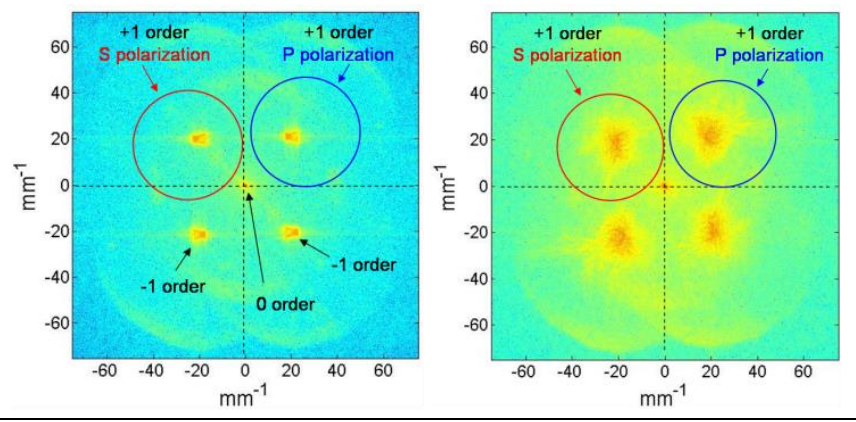

Fig. 5. 2D FFT spectra of interferograms recorded without flow (reference, left) and with flow (measurement, right).

For each $S$ and $P$ 2D Fourier spectra, the modulo $2 \pi$ phase maps for the reference and measurement interferograms are computed. Fig. 6 shows the phase difference obtained between the measurement and the reference for the polarization $S$ (left) and $P$ (right). The white circles indicate the areas where the phase exhibits singularities. They are located on the upper part of the $S$ polarization map and on the lower part of the P polarization map.

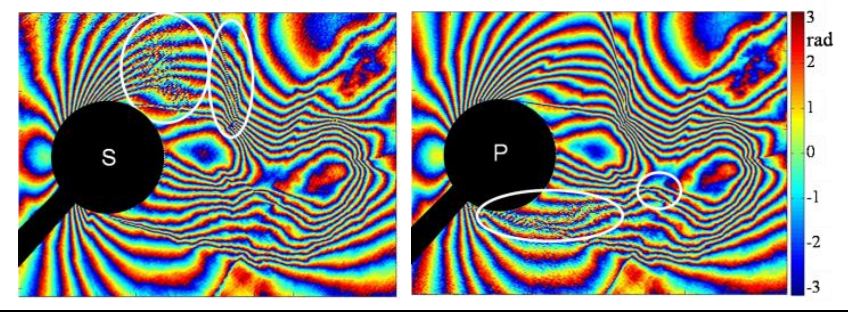

Fig. 6. Modulo $2 \pi$ phase difference maps (S and P polarization).

The unwrapping of modulo $2 \pi$ phase maps of Fig. 6 is presented in Fig. 7. The Herraez's algorithm which consists in determining a criterion of reliability for each pixel was used [17]. The criterion is equal to the inverse of the second difference with the neighboring pixels and the phase unwrapping can be operated following a non continuous path. The more reliable the pixels, the lower their second difference. An unwrapping path is defined by looking at the value of the reliability of the edges. The edges are stored in an array and sorted by value of reliability. The edges with a higher reliability are solved first. As seen in Fig. 7, the lower part of the S polarization image and the upper part of the $\mathrm{P}$ polarization image 
are correctly unwrapped and the phase singularities can be clearly seen in the white circles.

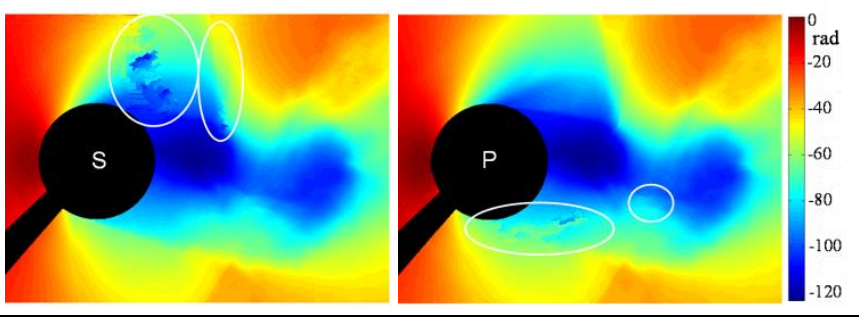

Fig. 7. Unwrapped phase difference maps (S and P polarization).

The fusion of these two phase maps yields an error-free phase map. The processed phase difference shown in Fig. 8 is obtained by taking the lower part of the phase map calculated with $\mathrm{S}$ polarization image and the upper part of the phase map calculated with $\mathrm{P}$ polarization image. Between both phase maps, in areas without phase shifts or where the phase varies continuously (for instance, at the cylinder nose), a phase difference equivalent to a piston phase which can be determined by comparing, at a given pixel, the phase found in the S polarization map and those given by the $\mathrm{P}$ polarization map. Then, the correction of piston phase is numerically applied for the selected area or for all half of the map. As consequence, the shock waves, the detachment zones and shear layers are very well restored and the phase map no longer shows any residual phase singularities.

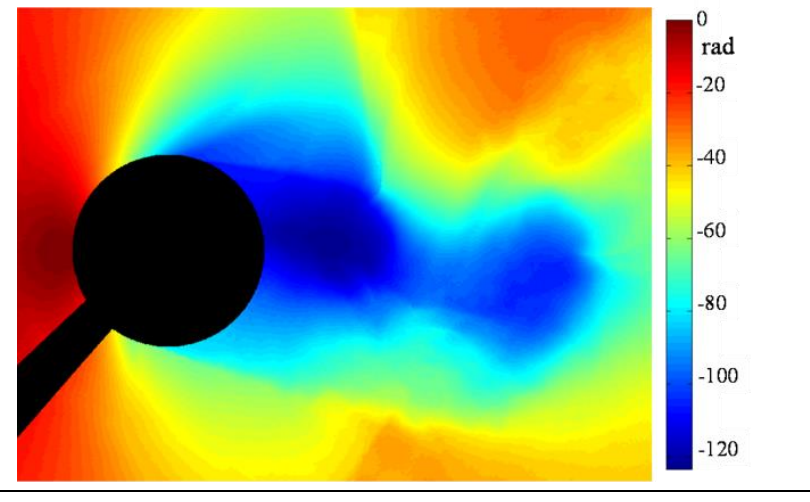

Fig. 8. Fusion of the phase maps from $S$ and P polarization data.

Note that the mixing of the two polarization images could be carried out by taking into account the highest values of the reliability factor on each modulo $2 \pi$ phase map [17]. From a point of view of Fluids Mechanics, a phase variation $\Delta \phi$ of about 120 radians between the stagnation point (just at the nose of the cylinder) and the center of the first vortex downstream the cylinder can be measured from the experimental results. The dimensionless gas density can be calculated from the phase difference $\Delta \phi$ according the following relationship:

$$
\frac{\rho}{\rho_{o}}=1-\left(\frac{\rho_{s}}{\rho_{o}} \frac{\lambda \Delta \phi}{2 \pi e K}\right)
$$

where $\rho_{s}$ is the standard gas density computed at 1 atmosphere and $0^{\circ} \mathrm{C}, \rho_{o}$ the stagnation gas density, $\lambda$ the wavelength of the interferometer, $e$ the width of the test section and $K$ the GladstoneDale constant $\left(\mathrm{K}=296 \times 10^{-6}\right)$. It follows that the gas density in the first vortex exhibits a strong decrease down to $90 \%$ of $\rho_{0}$. In the second vortex, the measured gas density is near to $80 \%$ of $\rho_{\text {o. }}$ A lot of interferograms have been analyzed in order to evaluate the axial evolution of the gas density along $x$ inside the vortex cores. Two phases have been identified behind the cylinder of diameter $D$. For $0.5<x / D<1$, the vortices are in a formation or agglomeration phase because the gas density decreases at their center. Then, when $\mathrm{x} / \mathrm{D}>1$, the vortices enter a phase of dissipation because the gas density increases again at their centers. It remains to compare the experimental results with the results yielded by Computational Fluid Dynamic codes.

As conclusion, dual-reference digital holographic interferometry using Wollaston prism is ideally suited for analyzing high refractive index encountered in transonic and supersonic flows.

Funding. The French National Research Agency (ANR) (ANR-14ASTR-0005).

\section{References}

1. F. Durst, A. Melling, and J.H. Whitelaw, Principles and Practice of Laserdoppler Anemometry (Academic Press, London, 1976).

2. R.J. Adrian and J. Westerweel, Particle image velocimetry (Cambridge University Press, 2011).

3. J.M. Desse, Advanced Holography-Metrology and Imaging, I. Naydenova, ed. (InTech, 2011) pp. 3-28.

4. J.M. Desse, P. Picart, and P. Tankam, Opt. Lasers Eng. 50, 18 (2012).

5. P. Tankam, Q. Song, M. Karray, J.C. Li, J.M. Desse, and P. Picart, Opt. Lett. 35, 2055 (2010).

6. G. Rajshekhar, S.S. Gorthi, and P. Rastogi, Appl. Opt. 50, H16 (2011).

7. J. Kühn, T. Colomb, F. Montfort, F. Charrière, Y. Emery, E. Cuche, P. Marquet, and C. Depeursinge, Opt. Express, 15, 7231 (2011).

8. A. Khmaladze, M. Kim, and C.M. Lo, Opt. Express, 16, 10900 (2008).

9. D. Khodadad, E. Amer, P. Gren, E. Melander, E. Hällstig, and M. Sjödahl, Proc. SPIE 9660, 96601E1 (2015).

10. D. Barada, T. Ochiai, T. Fukuda, S. Kawata, K. Kuroda, and T. Yatagai, Opt. Lett. 37, 4528 (2012).

11. T. Ochiai, D. Barada, T. Fukuda, Y. Hayasaki, K. Kuroda, and T. Yatagai, Opt. Lett. 38, 748 (2013).

12. H.C. Lee, S.H. Kim, and D.S. Kim, J. Opt. Korea 14, 363 (2010).

13. D.G. Abdelsalam, R. Magnusson, and D. Kim, Appl. Opt. 50, 3360 (2011).

14. D. Beghuin, E. Cuche, P. Dahlgren, C. Depeursinge, G. Delacrétaz, and R.P. Salathé, Electron. Lett. 35, 2053 (1999).

15. T. Colomb, P. Dalhgren, D. Beghuin, E. cuche, P. Marquet, and C. Depeursinge, Appl. Opt. 41, 27 (2002).

16. T. Tahara, Y. Awatsuji, Y. Shimozato, T. Kakue, K. Nishio, S. Ura, T. Kubota, and O. Motaba, Opt. Lett. 36, 3254 (2011).

17. M.A. Herraez, D. Burton, M.J. Lalor and A. Gdeisat, Appl. Opt. 41, 7437 (2002). 